\title{
Chronic Corticosterone Shifts Effort-Related Choice Behavior in Y-Maze
}

\author{
Prachi Srivastava \\ Written under the supervision of Dr. Benjamin Samuels
}

\begin{abstract}
Major depressive disorder (MDD) affects more than 16.1 million American adults (about 6.7\% of the U.S. population age 18+) in a given year (ADAA). Understanding how chronic stress impacts decision-making may allow us to help people suffering from MDD receive the most suitable antidepressant treatment given their behavioral tendencies in reward and motivational processing. In these experiments, our objective is to characterize effort-related choice tasks using chronic stressors in mice. To study this topic, we take advantage of the well-validated homology between corticosterone (CORT) in rodents and cortisol in humans to induce mood disorders such as MDD and chronic stress in mice. Effort-related choice tasks have been characterized using stressors in rats, but the stressors have not been chronic and have not been characterized in mice. The results of these experiments would be a better foundation for research involving antidepressant treatment experiments on mice. The experimental group was administered CORT in their drinking water throughout all experiments. Then, both the control and experimental groups were tested in a Y-Maze barrier task to demonstrate the behavioral effects of CORT. The high reward (HR) arm of the $Y$-Maze contained a reward of four food pellets, which required high effort to obtain. The low reward (LR) arm of the Y-Maze contained a reward of two food pellets, which required less effort to obtain Animals chronically exposed to CORT displayed a stronger preference for low-effort, low-reward choices than control subjects. The results suggest that chronic CORT may reduce motivation to work for a highly rewarding reinforcer when a less rewarding reinforcer is available.
\end{abstract}

\section{Introduction}

Motivation is a critical aspect of reward-related behaviors and is affected by mood disorders such as MDD (Schrader 1997). An important component of motivation is that it initiates and maintains goal-directed behaviors requiring persistent effort. This activating component of motivation can be specifically assessed in preclinical experimental models of depression using effort-related choice tasks (Salamone, et al. 1994).

Studies conducted by Belzung, et. al (2011) outline the criteria for animal models of psychiatric disorders, particularly in anxiety and depressive disorders, which are the focus of this study. These criteria include mechanistic validity, face validity, and predictive validity. Mechanistic validity indicates the extent to which the cognitive or biological mechanisms 
underlying stress disorders (such as stress hormone dysregulation) are similar in humans and the model organism. Face validity indicates the extent to which either the observable behavior (such as anhedonia) or the biological outcomes (such as elevated corticosterone, or CORT) are analogous in humans and the model organism. Finally, predictive validity indicates the extent to which a triggering factor will induce similar disorder-related outcomes in humans and the model organism, and whether the effects of treatments on the model organism mirror those on humans. The relevance of this framework is later discussed regarding various animal models of depression.

Effort-related choice behaviors are behaviors in which a subject chooses between a highly rewarding reinforcer that requires high effort to obtain and a less rewarding reinforcer that requires significantly less effort to obtain. The behavioral test discussed in this study is a Yshaped barrier maze task. In the Y-Maze barrier task, the rodents may choose to climb over a wire-mesh barrier to acquire four food pellets in the HR arm of the Y-Maze, versus traversing the LR arm with no barrier to acquire two food pellets (Yohn, et al. 2015). This behavioral task is used to determine whether a rodent is motivated to exert effort for a highly rewarding reinforcer or if the rodent will shift its preference to the reward that requires significantly less work to consume. The effort-related choice tasks in rodents can be seen as analogous to effort-expenditure tasks in humans (Belzung and Lemoine 2011). Due to their comparability, effort-related choice tasks provide a valid and translatable behavioral assessment for studying motivational processes in rodents.

Salamone et al. (2003) suggest that drugs that interfere with dopamine transmission such as CORT alter the rewarding aspects of primary reinforcers such as food. CORT is a stress hormone analogous to human cortisol and is the major output of the hypothalamuspituitary-adrenal axis, which is hyperactive in both humans with MDD and in rodent models of chronic stress. Chronic CORT has been shown to induce negative valence behaviors such as anxiety and impair positive valence behaviors such as reward processing (Dieterich 2019). Therefore, CORT was used to ensure mechanistic, face, and predictive validity. The effects of chronic CORT administration on effort-related choice behavior has not been characterized. Since stress-induced mood disorders such as depression involve deficits in reward processing and motivation, further study of how chronic stress influences effortrelated choice behavior is needed.

We hypothesized chronic CORT would reduce motivation for the high effort/high reward choices and shift responding to the less effort/less reward choices. To test this hypothesis, mice were chronically administered CORT and then tested in a Y-maze barrier task.

\section{Methodology}

\section{Control and experimental populations}

There were two groups of male mice: the control group (CG) mice $(n=6)$ and the experimental group (EG) mice $(n=8)$. The CG and EG mice were treated with $\beta$-cyclodextrin 
dissolved in their drinking water at a dose of $24 \mathrm{mg} / \mathrm{kg} /$ day. $\beta$-cyclodextrin was used to promote the subjects' drinking behaviors and increase consumption of the drinking water containing the dissolved CORT (David et al., 2009). The EG mice were treated with a dose of $5 \mathrm{mg} / \mathrm{kg} /$ day of CORT, which was chosen based on previous studies (David et al., 2009). EG mice were administered CORT during the four weeks prior to the experiment as well as throughout the experiment.

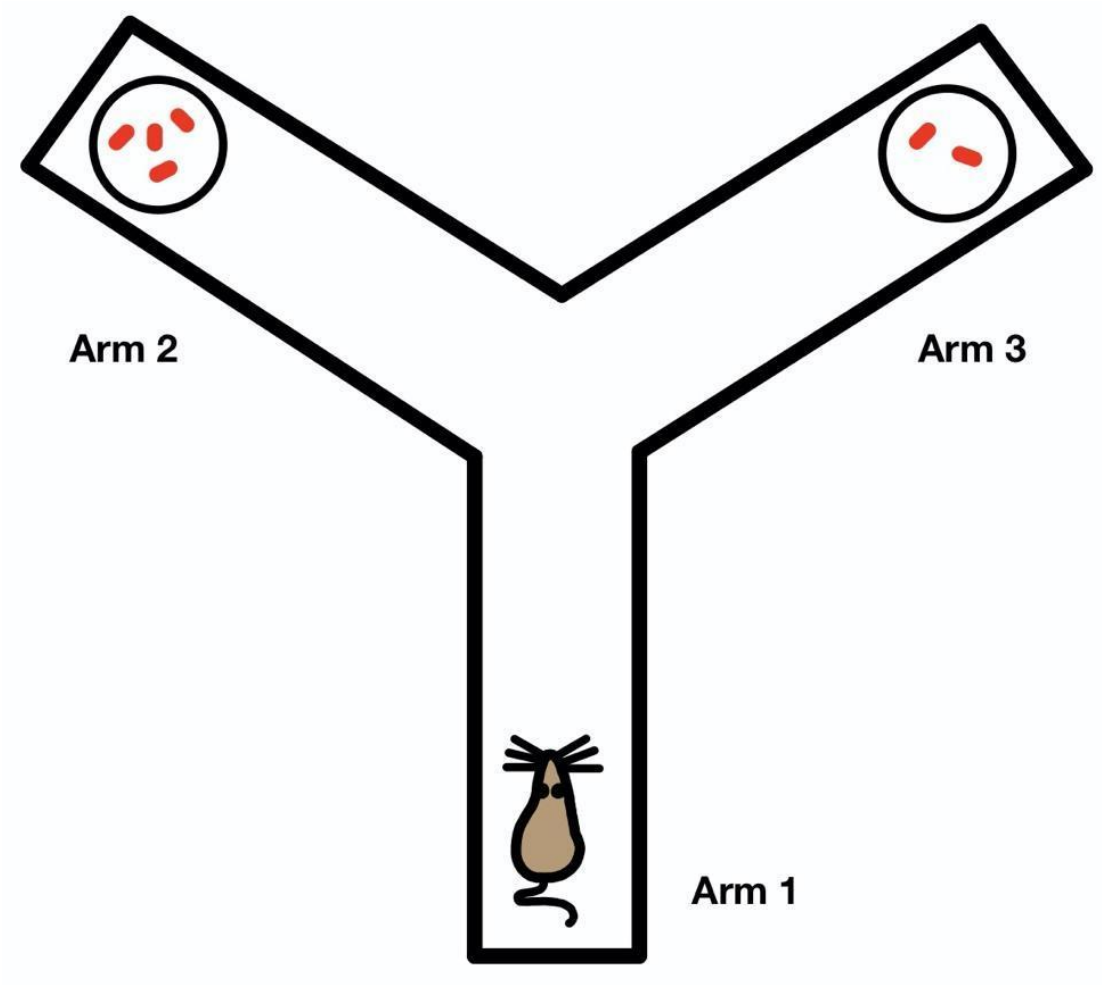

Figure 1. Y-Maze Setup. The Y-Maze consisted of a start box (Arm 1) where mice were placed to begin each trial. When a Plexiglas barrier was removed, the mouse could traverse the Y-Maze and enter either of the two arms. The high reward arm (Arm 2) side contained four pellets, and the low reward arm (Arm 3) contained two pellets. After training, progressively taller barriers were introduced into Arm 2 to force an effort-related choice between arms. Arm 2 and Arm 3 were counterbalanced across mice such that for half of the subjects, Arm 2 was opposite to what is indicated in this schematic.

\section{Y-Maze design}

A mouse version of the $\mathrm{Y}$-Maze barrier task used with rats (Yohn et al., 2015) was implemented to assess the effect of chronic CORT on effort-related decision-making. To begin each trial, mice were placed in a start box (Arm 1) behind a Plexiglas barrier (Fig 1). When the Plexiglas barrier was removed, the mouse could traverse the Y-Maze. Once it moved towards the center of the maze, the mouse could choose between entering Arm 2 or Arm 3 (Fig 1).

\section{Mouse training on $Y$-Maze}

After being given treated water for four weeks, mice were trained in the $\mathrm{Y}$-Maze barrier choice task to determine if chronic CORT alters effort-related choice behavior. Mice were 
first habituated to the maze over the course of two days in two 10-minute sessions with unlimited food pellets in Arms 2 and 3 of the Y-Maze (Fig 1). These initial habituation trials allow the mice to navigate through and become more familiar with the Y-Maze.

Mice then completed an additional five habituation trials over the course of five days, which ended after they had entered both arms and consumed all available food pellets. In the HR arm, Arm 2, there was a food bowl containing four pellets. In the LR arm, Arm 3, there was a food bowl containing two pellets. This experimental setup is outlined in Figure 1. The mice had free access to the entire maze during the habituation sessions, and no barriers were present in the arms. This was done to allow the mice to become acclimated to the maze and the differences in reward availability.

After habituation, mice completed two days of forced-choice sessions. These sessions included ten forced-choice trials where each arm of the maze was blocked off in alternating trials. These trials further acclimate the animals to the differences in reward between the HR and LR arms. Mice then were trained with five days of free-choice sessions, which began with two forced-choice trials followed by 10 free-choice trials. The free-choice sessions began with two forced-choice trials to serve as a reminder of the Y-Maze's layout at the start of each day. This accounts for any loss of learning in between trials. Following the two forced-choice trials, there were 10 free-choice trials. Each free-choice trial began with the mouse in the start box in Arm 1 where it had free access to choose between the HR and LR arms. In these sessions, there was no barrier placed in the $Y$-maze. Once the mouse entered one of the two arms, the entrance of that arm was blocked off with a barrier. The mouse then had one minute to consume the pellets before it was removed and returned to its home cage. Mice were trained in these free-choice sessions until they reached the criterion of $70 \%$ accuracy in choosing the HR arm. While the CG mice took 2-3 free-choice sessions to reach the criterion, the EG mice took all 5 days of free-choice sessions to reach the criterion. Thus, both groups reached the criterion prior to progressing to the following sessions.

In the following sessions, a barrier was added to the HR arm, requiring high effort since the mouse had to climb over a barrier before it could reach the food bowl. In Arm 3, no barrier was added; thus, reaching the food bowl required significantly less effort. The barriers used were 10,15 , or $20 \mathrm{~cm}$ tall wire-mesh barriers. Mice completed three days of testing with the $10 \mathrm{~cm}$ barrier in the HR arm, three days with the $15 \mathrm{~cm}$ barrier, and three days with the 20 $\mathrm{cm}$ barrier. Upon completing all test sessions, mice underwent three control sessions where $10 \mathrm{~cm}$ barriers were present in both HR and LR arms. This was done to test if CORT administration affected reward discrimination or the ability to climb over the barriers.

\section{Data Analysis}

A two-way ANOVA was used to examine the effect of CORT administration on HR arm selection in the multiple $Y$-Maze conditions described in the methods (Fig. 2). 


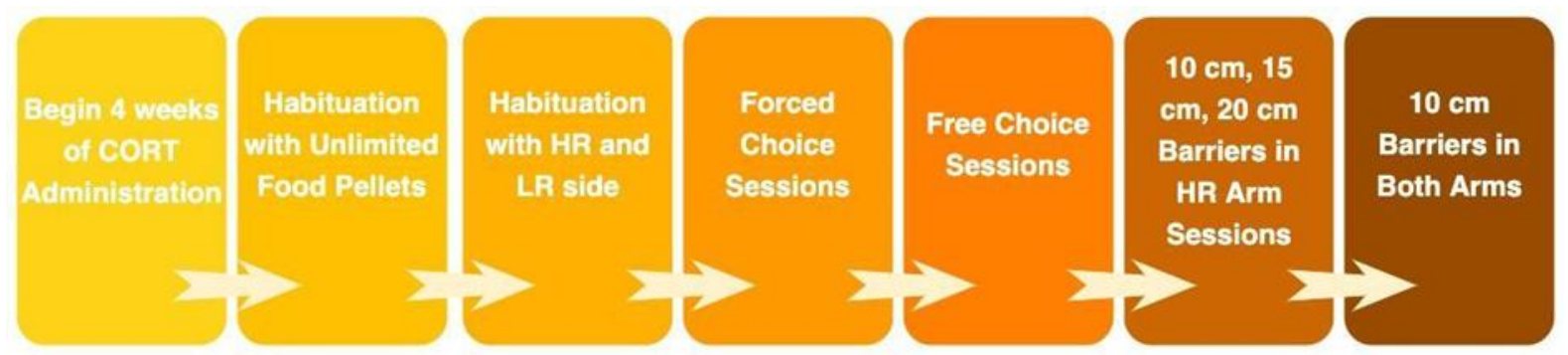

Figure 2. Experimental Timeline. After four weeks of CORT and $\beta$-cyclodextrin administration to the EG $(n=8)$ or $\beta$-cyclodextrin only to the CG $(n=6)$, mice were habituated to the maze over the course of two days with unlimited food pellets in the arms. Mice then completed an additional five days of habituation, with one arm always containing four pellets (Arm 2) and the other two pellets (Arm 3). After habituation, mice completed two days of forced-choice sessions and then were trained in five days of free-choice sessions with the HR and LR. Then, a barrier was added on Arm 2. Mice completed three days of testing with the $10 \mathrm{~cm}$ barrier in Arm 2, followed by three days with a $15 \mathrm{~cm}$ barrier, followed by three days with a $20 \mathrm{~cm}$ barrier, and lastly by a discrimination test with a $10 \mathrm{~cm}$ barrier in both arms.

\section{Results}

For the results to be significant the $p$-value must be $<0.001$. Using Bonferroni's multiple comparisons test (Bonferroni 2013), results suggest that CORT reduced HR arm selection when there was a $15 \mathrm{~cm}$ barrier $(p<0.001)$ or a $20 \mathrm{~cm}$ barrier $(p<0.001)$ placed in the HR arm. Importantly, there was no effect of CORT on HR arm selection without a barrier in the HR arm $(p=0.149)$, with a $10 \mathrm{~cm}$ barrier in the HR arm $(p=0.779)$, and when there were 10 $\mathrm{cm}$ barriers in both arms of the maze $(\mathrm{p}>0.999)$.

Therefore, chronic CORT shifts preference from HR to LR when greater effort is required to obtain the high reward, as HR arm selection was reduced with the 15 and $20 \mathrm{~cm}$ high barriers. CORT did not influence arm selection in trials without barriers or with $10 \mathrm{~cm}$ barriers in both arms compared to the CG. The results suggest that chronic CORT reduced $\mathrm{HR}$ arm choice in the Y-Maze when more effort was required to obtain the four food pellets, shifting preference to the LR arm where only two pellets were available.

\section{Discussion}

We used a Y-Maze barrier choice task to assess the effect of chronic CORT on effort-related choice behavior in mice. Effort-related choice behaviors have not been well-characterized in mice in other research (Cagniard et al. 2012). Also, no studies have assessed the effect of chronic CORT on effort-related decision-making. Many previous studies using chronic CORT have modeled negative valence behavior tests that induce stress, anger, or fear. These tests include forced swim tests that force rodents to avoid drowning. Negative valence tests such as the forced swim test are not well translatable to humans. However, the use of chronic CORT in positive valence behavior tests have been less well-characterized in animal models of depression. Positive valence behavior tests are tasks that induce pleasure or happiness. These include effort related choice tests that assess rodents' preference for higher rewards that require high effort versus lesser rewards that require less effort. These tests are more 
effective for testing behaviors using chronic CORT because the animal models for these behaviors are more translatable to human models of positive valence behaviors.

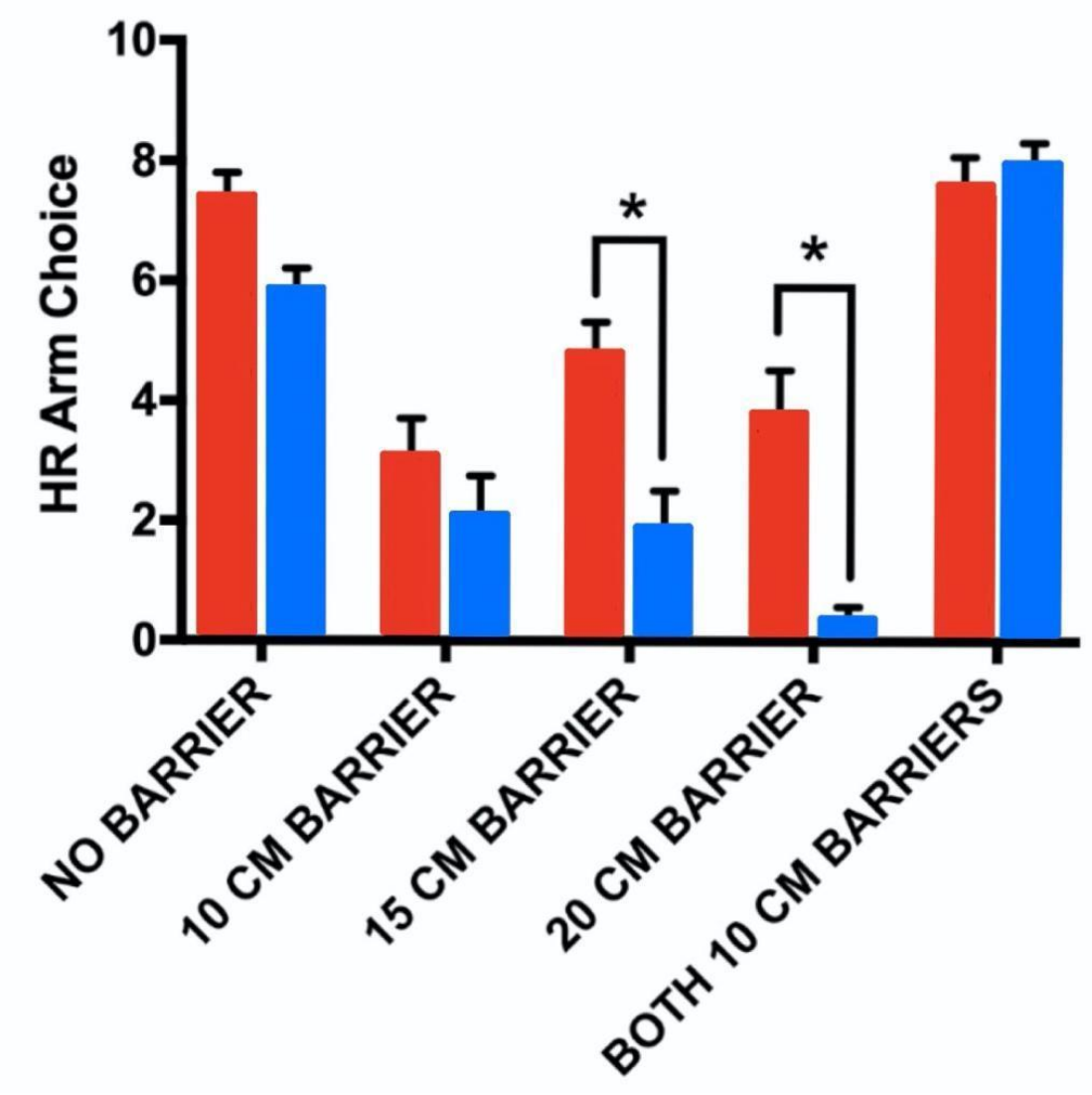

Control

Experimental

Figure 3. Effect of CORT on HR Arm Selection in Multiple Y-Maze Conditions. A two-way repeated measures ANOVA indicates a significant main effects of $Y$-Maze session, $(F(4,48)=76, p<0.001)$, and CORT administration, $(F(1,12)=15, p=0.002)$, as well as a significant interaction, $(F(4,48)=$ $6.7, \mathrm{p}<0.001)$. Comparisons indicate that CORT administration reduced HR arm selection relative to Control administration for $15 \mathrm{~cm}$ barrier $(p<0.001)$ and $20 \mathrm{~cm}$ barrier $(p<0.001)$ test sessions.

Thus, chronic CORT shifts preference from HR to LR when greater effort is required to obtain the high reward. CORT did not influence arm selection compared to the CG in trials without barriers or with 10 $\mathrm{cm}$ barriers in both arms. The results suggest that chronic CORT reduced HR arm preference in the $Y-$ Maze when more effort was required to obtain the high reward and shifted choice to the LR arm with the lesser reward.

The results of our experiments suggest that chronic CORT alters effort-related choice behavior in the $\mathrm{Y}$-maze barrier task in mice. Chronic CORT administration may reduce motivation to work for and obtain a highly rewarding reinforcer when a lesser reinforcer is freely available. This is the first study to examine the effect of chronic stress on effortrelated choice behaviors in mice. Future studies might compare behavioral changes in male and female mice to obtain more comprehensive results. Additionally, since chronic CORT administration may impair spatial memory-which could impact selection of the HR or LR arm independently of the effort-related choice-the relationship between CORT administration and spatial memory is another area for future research. 
Since chronic mood disorders such as depression are highly prevalent, we hope that this research will ultimately be useful for identifying novel treatments, determining whether patients will respond to treatments, and developing personalized treatment strategies.

\section{References}

Aberman JE, Salamone JD. Nucleus accumbens dopamine depletions make rats more sensitive to high ratio requirements but do not impair primary food reinforcement. Neuroscience. 1999; 92:545-552.

Anxiety and Depression Association of America (ADAA). (n.d.). Retrieved from https://adaa.org/about-adaa/press-room/facts-statistics

Belzung, C., \& Lemoine, M. (2011). Criteria of validity for animal models of psychiatric disorders: focus on anxiety disorders and depression. Biology of mood $\&$ anxiety disorders, 1(1), 9. doi:10.1186/2045-5380-1-9

Bonferroni's Method. NIST/SEMATECH e-Handbook of Statistical Methods, 30 Oct. 2013 , www.itl.nist.gov/div898/handbook/prc/section4/prc473.htm.

Cagniard, B., P. D. Balsam, D. Brunner and X. Zhuang (2006). "Mice with chronically elevated dopamine exhibit enhanced motivation, but not learning, for a food reward." Neuropsychopharmacology 31(7): 1362-1370.

David, D. J., B. A. Samuels, Q. Rainer, J.-W. Wang, D. Marsteller, I. Mendez, M. Drew, D. A. Craig, B. P. Guiard and J.-P. J. N. Guilloux (2009). "Neurogenesis-dependent andindependent effects of fluoxetine in an animal model of anxiety/depression." 62(4): 479-493.

Dieterich, A., Srivastava, P., Sharif, A. et al. Chronic corticosterone administration induces negative valence and impairs positive valence behaviors in mice. Trans/ Psychiatry 9, 337 (2019). https://doi.org/10.1038/s41398-019-0674-4

Eerde, Wendelien. (2015). Motivation and Reward Systems. 10.1002/9781118785317.weom060146.

Salamone, J. D., M. Correa, S. Mingote, S. J. J. o. P. Weber and E. Therapeutics (2003). "Nucleus accumbens dopamine and the regulation of effort in food-seeking behavior: implications for studies of natural motivation, psychiatry, and drug abuse." 305(1): 1-8.

Salamone, J. D., M. S. Cousins and S. Bucher (1994). "Anhedonia or anergia? Effects of haloperidol and nucleus accumbens dopamine depletion on instrumental response selection in a T-maze cost/benefit procedure." Behav Brain Res 65(2): 221-229.

Schrader, G. J. C. p. (1997). "Does anhedonia correlate with depression severity in chronic depression?" 38(5): 260-263.

Yohn, S. E., C. Thompson, P. A. Randall, C. A. Lee, C. E. Muller, Y. Baqi, M. Correa and J. D. Salamone (2015). "The VMAT-2 inhibitor tetrabenazine alters effort-related decision making as measured by the T-maze barrier choice task: reversal with the adenosine A2A antagonist MSX-3 and the catecholamine uptake blocker bupropion." Psychopharmacology (Berl) 232(7): 1313-1323. 
Aresty Rutgers Undergraduate Research Journal, vol. 1, issue 1, Spring 2020

\section{Acknowledgements}

This work was funded by NIMH R01 MH1 12861 (BAS). 